\title{
Papeterie et recyclage dans les villages de métier
}

La fin du modèle de production du delta du fleuve Rouge? (Vietnam)

Papermaking and Recycling in Craft Villages. The End of a Production Model?

(Red River Delta, Vietnam)

\section{Sylvie Fanchette}

\section{(2) OpenEdition \\ Journals}

Édition électronique

URL : https://journals.openedition.org/tc/7901

DOI : $10.4000 /$ tc. 7901

ISSN : 1952-420X

Éditeur

Éditions de l'EHESS

Édition imprimée

Date de publication : 31 octobre 2016

Pagination : 198-201

ISBN : 9782713225291

ISSN : $0248-6016$

Référence électronique

Sylvie Fanchette, «Papeterie et recyclage dans les villages de métier», Techniques \& Culture [En ligne], 65-66 | 2016, mis en ligne le 14 novembre 2016, consulté le 29 septembre 2022. URL : http:// journals.openedition.org/tc/7901; DOI : https://doi.org/10.4000/tc.7901 


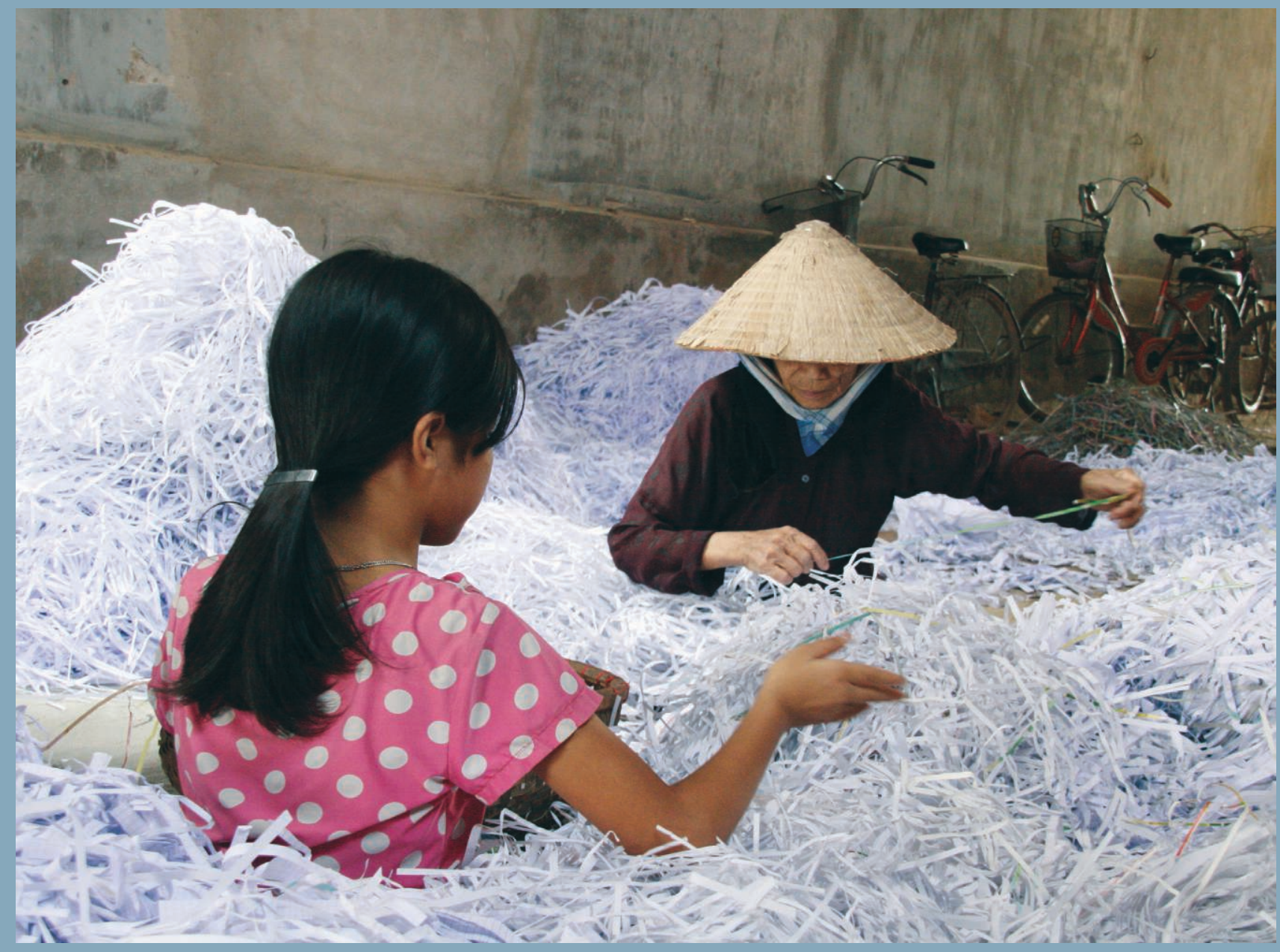




\section{Papeterie et recyclage dans les villages de métier}

\section{La fin du modèle de production du delta du fleuve Rouge? (Vietnam)}

Dans le delta du fleuve Rouge, le recyclage des matériaux est une pratique ancienne. Les savoirfaire de la population ont été largement conviés lors des périodes de restriction liées aux deux guerres anticoloniales et lors de l'embargo commercial en provenance des pays occidentaux.

Lartisanat occupe depuis plus de mille ans plusieurs centaines de milliers de villageois. En 2006, on comptait plus de mille villages de métier dans le delta du fleuve Rouge. Spécialisés dans des activités très variées, les villages sont organisés en ensembles, au sein desquels les liens techniques et familiaux et les réseaux économiques constituent le ciment social. Situés à l'interface des commerçants de déchets et objets usés et des villages de métier, des villages d'artisans se sont spécialisés dans le tri et le recyclage de matières premières prêtes à l'usage.

La papeterie date de plusieurs siècles et a intégré en amont de la longue chaîne opératoire les collecteurs et les trieurs. Avec les changements des modes de consommation, la scolarisation massive et l'augmentation et la diversification de la production, la papeterie s'est mécanisée et a complètement changé son rapport aux matières premières.

Dans le groupe de la papeterie de Phong Khê (Province de Bac Ninh), plusieurs techniques de production du papier cohabitent : la fabrication manuelle avec levage de la feuille à partir d'une pâte faite d'écorces de do (Wikstroemia balansae Gilg.) ; celle manuelle du papier de faible qualité à partir des déchets de papier; la fabrication mécanisée sur des chaînes de cuisson dans des usines comptant plusieurs dizaines d'ouvriers. Dans ces usines, on utilise à la fois des résidus de papier de qualité et de la cellulose importée. Des entreprises mécanisées ont émergé avec l'ouverture économique des années 1980 et occupent des «niches» de production de qualité intermédiaire. De l'amont (la collecte) à l'aval (la production, puis la découpe ou l'impression) de la chaîne opératoire, l'évolution des techniques ne s'est donc pas effectuée au même rythme. La collecte de déchets en ville reste toutefois très segmentée, saisonnière et informelle, et s'intègre difficilement dans la chaîne de production de la papeterie en cours d'industrialisation et de diversification. 
L’offre irrégulière des déchets de papier ne correspond plus à la demande d'entreprises modernes soumises à des impératifs de qualité, de standardisation et de délais de production.

L'ouverture économique et l'urbanisation des marges de Hà Nôi ont eu raison du système de production traditionnel de la papeterie. Très gourmande en eau comme en bois de chauffage pour les fours, la papeterie traditionnelle est victime de la concurrence de l'industrie depuis les années 1980 (Le Failler 2009). En 1994, l'État vietnamien interdit la fabrication des pétards, devenue trop dangereuse, et sonne le glas de la papeterie manuelle à Phong Khê, dont de nombreux papetiers vendaient leur papier de faible qualité aux fabricants de pétards.

En 2005, la production de papier de la province de Bac Ninh, dont fait partie le groupe de Phong Khê, représente 16,42\% du total produit dans le pays et occupe plus de trois mille personnes. En vingt ans, les papetiers de Phong Khê ont effectué un saut technologique impressionnant et ont augmenté leur volume de production qui atteint chaque année environ trente mille tonnes. De la production manuelle de giay do, ils se reconvertissent à la production mécanique de papier machine, papier toilette, papier kraft ou de carton, sur environ deux cent dix chaînes de moyenne ou grande envergure, certaines employant une centaine d'ouvriers. Les plus gros entrepreneurs se spécialisent dans le papier machine et le papier destiné à la fabrication des cahiers de qualité. Ils investissent dans des chaînes de cuisson de papier, agrandissent leur atelier, embauchent des ouvriers spécialisés recrutés à l'extérieur de la province et diversifient leur clientèle, entreprise complexe dans ce pays en transition vers l'économie de marché. Ils utilisent de la cellulose et des chutes de papier de qualité et diversifient leur approvisionnement en matière première. Ils ciblent

1. Entreprise de papeterie mécanisée (production de papier kraft).

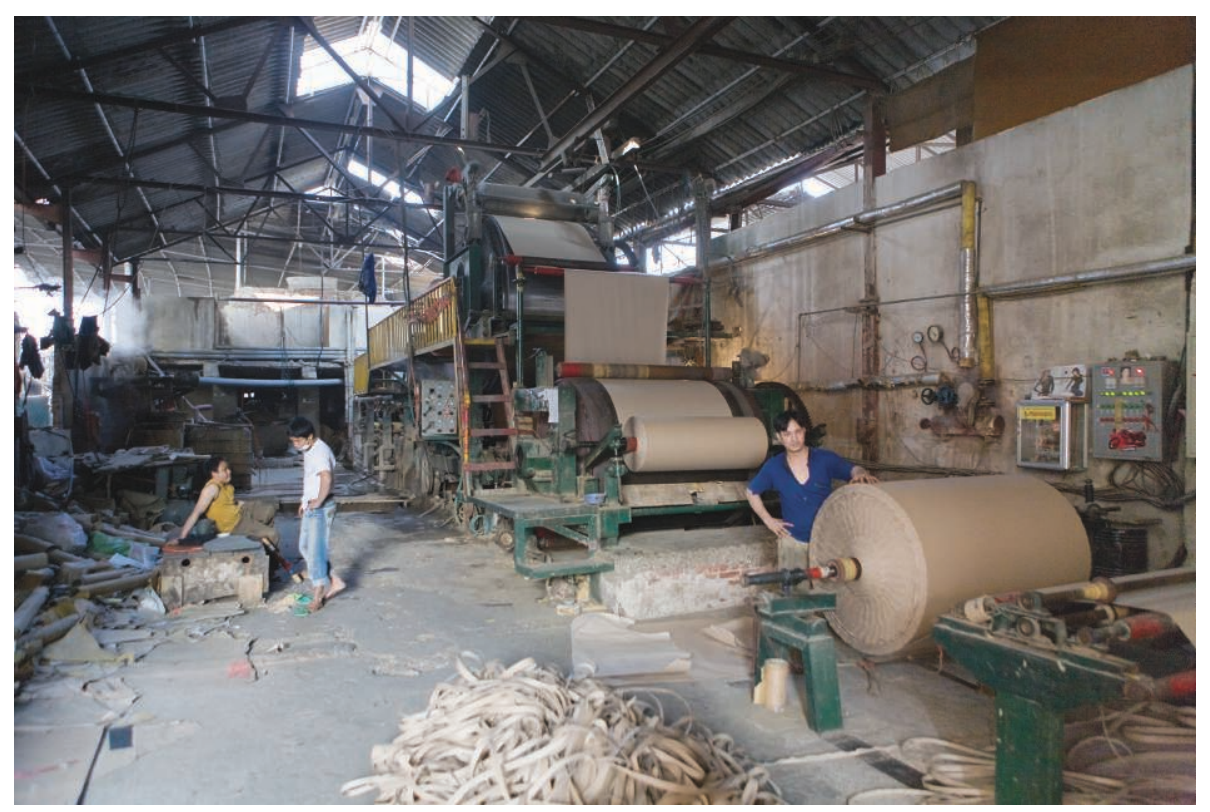


le marché domestique, et notamment celui du papier de qualité moyenne laissé par les grandes entreprises d'État subventionnées qui alimentent les marchés urbains en articles de qualité.

Toutefois, seules les entreprises disposant d'entrepôts peuvent stocker de larges volumes de matière à recycler. Les plus mécanisées cherchent à améliorer la qualité de leurs produits et à diversifier leur offre pour pallier l'intense concurrence et la pression sur les coûts de production.

Le secteur informel à Hà Nôi recycle 22 \% des déchets produits par les habitants de la ville. Les villages de métier absorbent une grande partie des papiers, des métaux et des plastiques usagés et s'approvisionnent à $80 \%$ sur ce marché informel. Les déchets de papier représentent 28 \% du poids collecté par le secteur informel de Hà Nôi, en deuxième place après les bouteilles en plastique (40\%). À Hà Nôi, la collecte des déchets est très segmentée et hiérarchisée et se compose d'entreprises municipales (URENCO, Urban Environment Company) et d'un large secteur informel formé d'environ dix mille collectrices ambulantes (Mitchell 2006) dont nous rapportons le quotidien et l'économie dans l'article en ligne.

\section{En ligne}

Retrouvez l'article complet, sur revues.org, TechniquesECulture 65-66 « Réparer le monde. Excès, reste et innovation »: http://tc.revues.org.

\section{I'auteure}

Sylvie Fanchette est géographe, chargée de recherche à l'Institut de recherche pour le développement (IRD). Ses recherches portent sur le processus d'urbanisation in situ et l'industrialisation dans les zones rurales dans les deltas très peuplés d'Asie, et les relations villes-campagnes (migrations, innovation, changements sociaux) (Vietnam et Inde).

\section{Iconographie}

Image d'ouverture. Tri de chutes de papier à domicile. (c) Sylvie Fanchette

1. () François Carlet-Soulage NoiPictures

\section{Références}

Le Failler, P. 2009 «L'industrie du papier selon Oger », préface in H. Oger Technique du peuple annamite (1909). O. Tessier \& P. Le Failler éd. Hà Nôi : École française d'Extrême-Orient.

Mitchell, C.L. 2006 Recycling the City: The Impact of Urban Change on the Informel Waste-Recovery Trade in Hanoi, Vietnam. PhD, Department of Geography, University of Toronto.

\section{Pour citer cet article}

Fanchette, S. 2016 «Papeterie et recyclage dans les villages de métier. La fin du modèle de production du delta du fleuve Rouge (Vietnam)?», TechniquesECulture 65-66 «Réparer le monde. Excès, reste et innovation», p. 198-201. 